\title{
Quantum Mechanics/Molecular Mechanics Electrostatic Embedding with Continuous and Discrete Functions
}

\author{
G. Andrés Cisneros ${ }^{*}$, Jean-Philip Piquemal, and Thomas A. Darden ${ }^{*}$ \\ Laboratory of Structural Biology, National Institute of Environmental Health Sciences, Box 12233 \\ MD F0-08 Research Triangle Park (RTP), North Carolina 27709
}

Abstract

A quantum mechanics/molecular mechanics (QM/MM) implementation that uses the Gaussian electrostatic model (GEM) as the MM force field is presented. GEM relies on the reproduction of electronic density by using auxiliary basis sets to calculate each component of the intermolecular interaction. This hybrid method has been used, along with a conventional QM/MM (point charges) method, to determine the polarization on the QM subsystem by the MM environment in QM/MM calculations on 10 individual $\mathrm{H}_{2} \mathrm{O}$ dimers and a $\mathrm{Mg}^{2+}-\mathrm{H}_{2} \mathrm{O}$ dimer. We observe that GEM gives the correct polarization response in cases when the MM fragment has a small charge, while the point charges produce significant over-polarization of the QM subsystem and in several cases present an opposite sign for the polarization contribution. In the case when a large charge is located in the MM subsystem, for example, the $\mathrm{Mg}^{2+}$ ion, the opposite is observed at small distances. However, this is overcome by the use of a damped Hermite charge, which provides the correct polarization response.

The study of chemical reactions that take place in a small region of a large system, for example, enzyme reactions, is a challenging problem. This problem has been tackled by several approaches, one of which relies on combining quantum mechanics $(\mathrm{QM})$ and molecular mechanics (MM) methods (QM/ MM). ${ }^{1}$ In this approach, QM programs are used on the small part of the system involved in the chemical reaction, while a MM force field (FF) is used to treat the rest of the system. This approach has been successfully applied to simulate the mechanisms of many enzymes. ${ }^{2}$ However, current QM/MM methods rely on FFs that use simple point charges to represent the Coulombic contribution in the MM subsystem. These point charges have been shown to over-polarize the QM wave function when they are in close proximity to the QM subsystem. ${ }^{3}$ One way to correct this problem is by using a delocalized charge, such as a Gaussian charge distribution. ${ }^{4}$ Another possibility is to use methods based on frozen density, which rely on molecular orbitals. ${ }^{5}$

We have recently developed a FF based on density fitting methods called the Gaussian electrostatic model (GEM). GEM relies on the use of molecular electronic density to calculate the intermolecular interaction by separately evaluating each of its components, that is, Coulomb, exchange, polarization, and charge transfer. ${ }^{6,7}$ The molecular electronic density is reproduced by fitting the fragment density calculated with QM methods to an auxiliary basis set (ABS), which makes GEM considerably faster than frozen density approaches.

In this contribution, we present a nonbonded QM/MM implementation that interfaces the Gaussian $98^{8}$ program with GEM, an approach similar to frozen density methods. ${ }^{5}$ This initial

\footnotetext{
*To whom correspondence should be addressed. E-mail: cisnero1@ niehs.nih.gov; darden@niehs.nih.gov.

Supporting Information Available: QM/GEM implementation, damped charge formula, and results for 10 dimers with A1 and P1 ABS. This material is available free of charge via the Internet at http://pubs.acs.org.
} 
implementation takes into account only frozen core contributions (Coulomb and exchange) calculated by GEM (see Supporting Information). To our knowledge, this is the first QM/ MM implementation that represents the MM environment by a FF based not on point charges (or delocalized point charges) but rather on explicit modeling of the molecular electron density. Moreover, along with the greatly increased accuracy of electrostatic potentials, fields, and interaction energies, ${ }^{6,7}$ GEM provides the ability to include MM electron density determined at the same level of theory as that used for the QM region in the QM/MM calculation. Indeed, we have shown that GEM can model the QM theory level, in the sense that dimer interaction energies calculated with full QM and with GEM fitted at the same level of theory show smaller differences than the same energies calculated with full QM at different levels of theory. 6,7

We have tested this new QM/MM implementation on 10 individual water dimers studied by van Duijneveldt-van de Rijt et al., ${ }^{9}$ as well as the distance dependence for the canonical water dimer (structure 1 in the 10 dimers) and a $\mathrm{Mg}^{2+}{ }_{-} \mathrm{H}_{2} \mathrm{O}$ dimer. These results are compared with conventional QM/MM results (point charges).

In the present contribution, we are only interested in the polarization effect of the MM environment on the QM subsystem, therefore we have not included the QM/MM exchange (or $\mathrm{QM} / \mathrm{MM} \mathrm{vdW}$ for conventional QM/MM) in the results presented below. However, it is important to note that the remaining components (exchange, polarization, and charge transfer) are not negligible and account for a significant part of the total intermolecular interaction energy. 6,9

The polarization energy of the QM subsystem has been approximated in the following manner: First, the energy of the isolated molecule that corresponds to the monomer located in the QM subsystem is determined. Subsequently, this value is subtracted from the QM/MM energy of the dimer, which gives the electrostatic + polarization interaction on the QM subsystem. The final approximate polarization of the QM molecule is obtained by subtracting the constrained space orbital variation $(\mathrm{CSOV})^{10}$ electrostatic energy from the electrostatic + polarization interaction. This result is compared with its CSOV counterpart, which is calculated by relaxing the molecular orbitals of monomer A in the potential generated by monomer B, freezing the occupied, and excluding the virtual orbitals of the latter from the self-consistent field (SCF). The orbitals of monomer A are kept orthonormal to the occupied orbitals of monomer B. ${ }^{10}$

Note that the polarization value from the QM/MM calculation is only approximate compared with the CSOV result because the polarization contribution in the CSOV procedure is calculated using orthogonalized orbitals, which is impossible in frozen density approximations. ${ }^{11}$ It is also important to point out that, by calculating the polarization effect on the QM subsystem in this way, it is assumed that the electrostatic interaction is exact compared with $\mathrm{CSOV}$ and therefore all the error is in the polarization term.

All the fitted densities for GEM as well as the Merz-Kollman (MK) point charges have been obtained at the B3LYP/6-31G* level of theory. The g03 ABS used for the fit is the same as that in ref 12. The Hermite coefficients for the auxiliary basis, as well as the multipoles from the Hermite coefficients, were obtained from the calculations in ref 7. In all cases, the QM/ $\mathrm{MM}$ calculations were performed by placing one monomer in the QM and the other in the MM subsystems.

Table 1 shows the polarization response for the 10 water dimers calculated with CSOV and QM/MM with both GEM and regular point charges including TIP3P and MK charges. In all cases, the response was calculated for each monomer in the dimer. As can be seen, the QM/ GEM polarizations are in good agreement with the CSOV results, with average absolute errors below $0.07 \mathrm{kcal} / \mathrm{mol}$. On the other hand, average absolute errors around $0.5 \mathrm{kcal} / \mathrm{mol}$ or above are obtained from the calculations that employ the multipoles obtained from the Hermite 
coefficients (mult. g03) as well as with the TIP3P and MK charges. Moreover, in the case of the point charges, the sign for the polarization response is positive, indicating an unfavorable polarization interaction. This is most likely due to a lack of penetration effects, which are present when using Gaussian functions.

The results for the distance dependence for the water dimer are shown in Figure 1. In all cases, errors are observed at very close range (around $1.5 \AA$ ). For hydrogen bonding distances that can be seen in conventional MD calculations, around $2.5 \AA$ and above, GEM shows very good agreement with CSOV while the point charges have errors of over $5 \mathrm{kcal} / \mathrm{mol}$. As can be expected, both the continuous and discrete representations converge to the correct value at larger distances.

Figure 2 shows the results for the $\mathrm{Mg}^{2+}-\mathrm{H}_{2} \mathrm{O}$ dimer. When the $\mathrm{Mg}^{2+}$ cation is placed in the QM subsystem (Figure 2, top), the polarization response from GEM matches the CSOV results even at very close range, while the point charge model shows significant over-polarization. On the other hand, when the water molecule is in the QM subsystem (Figure 2, bottom), the point charge model performs better than GEM at close range and both converge to the correct value at medium (and long) range. This is due to the lack of exchange polarization, that is, since the orbitals of the QM subsystem cannot be orthogonalized with the GEM density, we cannot capture the exchange polarization contribution. 6,12 In this case, the perturbation of a +2 charge on the QM subsystem is too large to approximate with linear response which results in the errors from GEM at close range. Figure 2 (bottom) also shows the polarization results with a damped Hermite charge using a modified version of eq 7 of ref 11a (see Supporting Information); as can be seen, the results are significantly improved. Moreover, it is important to note that the closest $\mathrm{Mg}^{2+}-\mathrm{H}_{2} \mathrm{O}$ distance from the 1st peak of the radial distribution function is located around $2.1 \AA{ }^{13}$ at this distance, the results from GEM are in agreement with CSOV.

On the basis of these results, it is evident that, although point charges produce the correct polarization at long range on average, care needs to be taken when dealing with medium and close range interactions, even for nonbonded systems. Note that in conventional QM/MM methods, the remaining $\mathrm{QM} / \mathrm{MM}$ and $\mathrm{MM}$ terms may compensate, at least partly, for some of these errors.

In conclusion, we have presented a new $\mathrm{QM} / \mathrm{MM}$ implementation that uses GEM to represent the MM subsystem. For fragments with small charge, this FF provides the correct polarization response for the QM wave function in contrast to FFs based on point charges. For fragments with large charge (e.g., ions), a damping function can be used to overcome the over-polarization problem. This method also provides the ability to use matching levels of theory between the $\mathrm{QM}$ and MM subsystems. It is expected to be of use in QM/MM calculations where the correct polarization of the QM is required, for example, when a critical hydrogen bond interaction exists between a molecule located in the QM subsystem and another located in the MM environment.

\section{Supplementary Material}

Refer to Web version on PubMed Central for supplementary material.

\section{Acknowledgment}

This research was supported by the Intramural Research program of the NIH and NIEHS. Computing time from the Advanced Biomedical Computing Center, NCI-FCRDC is gratefully acknowledged. 


\section{References and Notes}

(1). Warshel A, Levitt M. J. Mol. Biol 1977;103:227-235. [PubMed: 985660]

(2). Kollman P, Kuhn B, Perakyla M. J. Phys. Chem. B 2002;106:1537-1542.

(3) (a). König P, Hoffman M, Frauenheim T, Cui Q. J. Phys. Chem. B 2005;109:9082-9095. [PubMed: 16852081] (b) Vreven T, Byun KS, Komáromi I, Dapprixh S, Montgomery JA Jr. Morokuma K, Frisch MJ. J. Chem. Theor. Comput 2006;2:815-826.

(4). Das D, Eurenius P, Billings EM, Sherwood P, Chatfield DC, Hodoscek M, Brooks BR. J. Chem. Phys 2003;117:10543-10547.

(5) (a). Wesolowski TA, Warshel A. J. Phys. Chem 1993;97:8050-8053. (b) Valderrama E, Wheatley RJ. J. Comput. Chem 2003;24:2075-2082. [PubMed: 14531060]

(6). Piquemal JP, Cisneros GA, Reindhardt P, Gresh N, Darden A. J. Chem. Phys 2006;124:104101. [PubMed: 16542062]

(7). Cisneros GA, Piquemal JP, Darden A. J. Chem. Phys. submitted for publication

(8). Frisch, MJ., et al. Gaussian 98. Gaussian Inc.; Pittsburgh, PA: 1998. revision A.8

(9) (a). Tschumper GS, Leininger ML, Hoffman BC, Valeev EF, Quack M, Schaffer HF III. J. Chem. Phys 2002;116:690-701. (b) van Duijneveldt-van de Rijdt JGCM, Mooij WTM, Duijneveldt FB. Phys. Chem. Chem. Phys 2003;5:1169-1180.

(10) (a). Bagus PS, Illas F. J. Chem. Phys 1992;96:8962-8970. (b) Piquemal JP, Marquez A, Parisel O, Giessner-Prettre C. J. Comput. Chem 2005;26:1052-1062. [PubMed: 15898112]

(11) (a). Piquemal JP, Gresh N, Giessner-Prettre C. J. Comput. Chem 2003;107:10353-10359. (b) Dułac M, Wesołowski TA. J. Chem. Phys 2006;124:164101. [PubMed: 16674123]

(12). Cisneros GA, Piquemal JP, Darden A. J. Chem. Phys 2005;123:044109. [PubMed: 16095348]

(13). Piquemal JP, Perera L, Cisneros GA, Pedersen LG, Darden TA. J. Chem. Phys. in press 


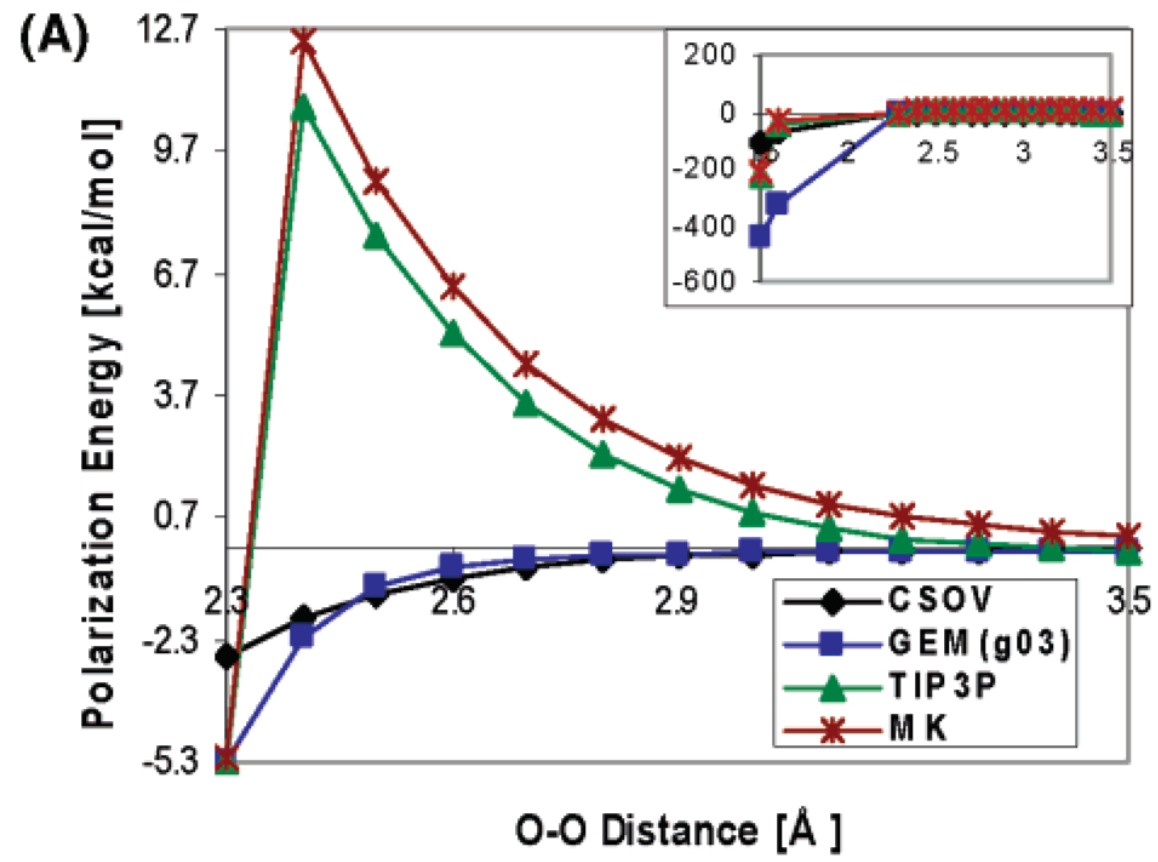

(B)

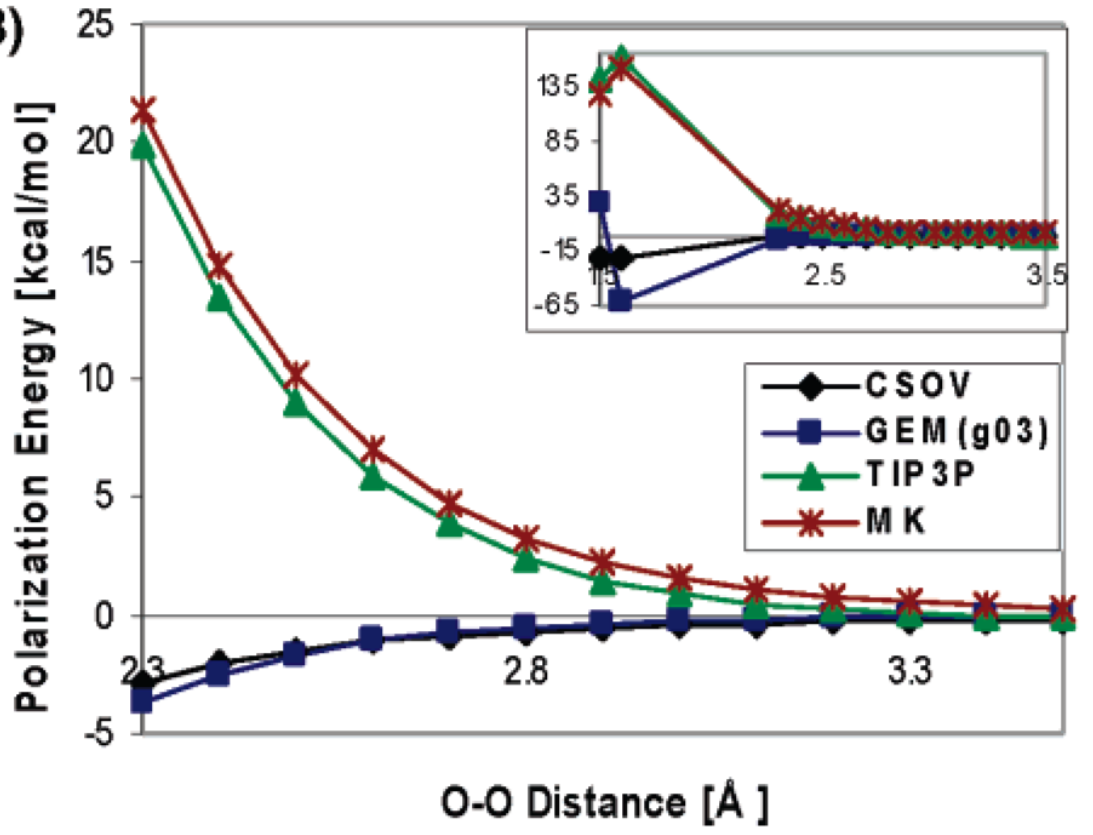

Figure 1.

Polarization for dimer 1 (molecule A, top; molecule B, bottom) for a range of distances. Inset shows a range from 1.5 to $3.5 \AA$. 
(A)

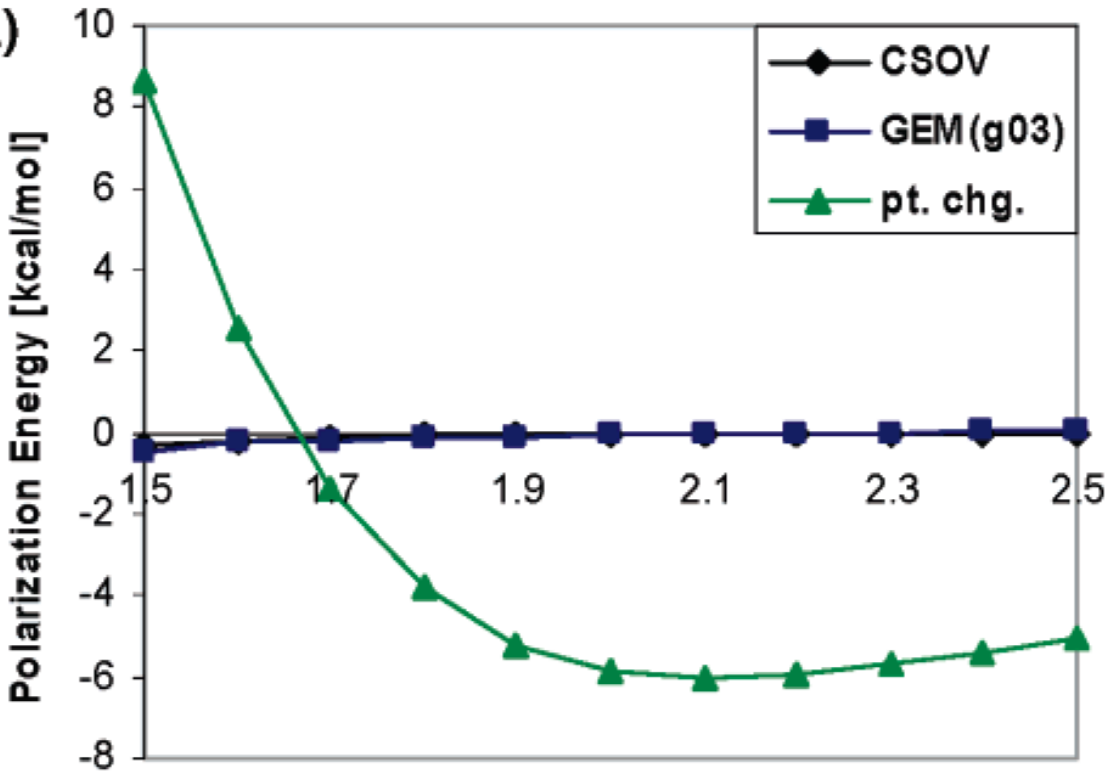

Mg-O Distance [A ]

(B)

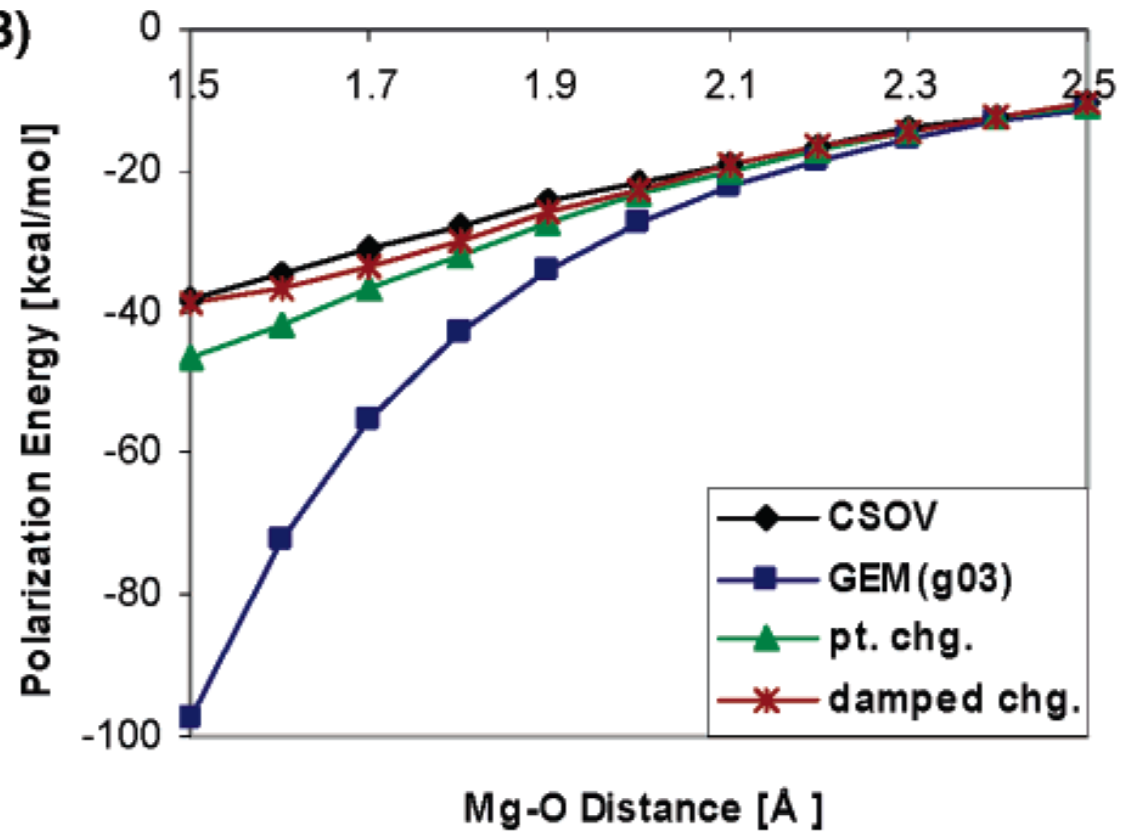

Figure 2.

Polarization for the $\mathrm{Mg}^{2+}-\mathrm{H}_{2} \mathrm{O}$ dimer $\left(\mathrm{Mg}^{2+}\right.$, top; $\mathrm{H}_{2} \mathrm{O}$, bottom) for a range of distances. 


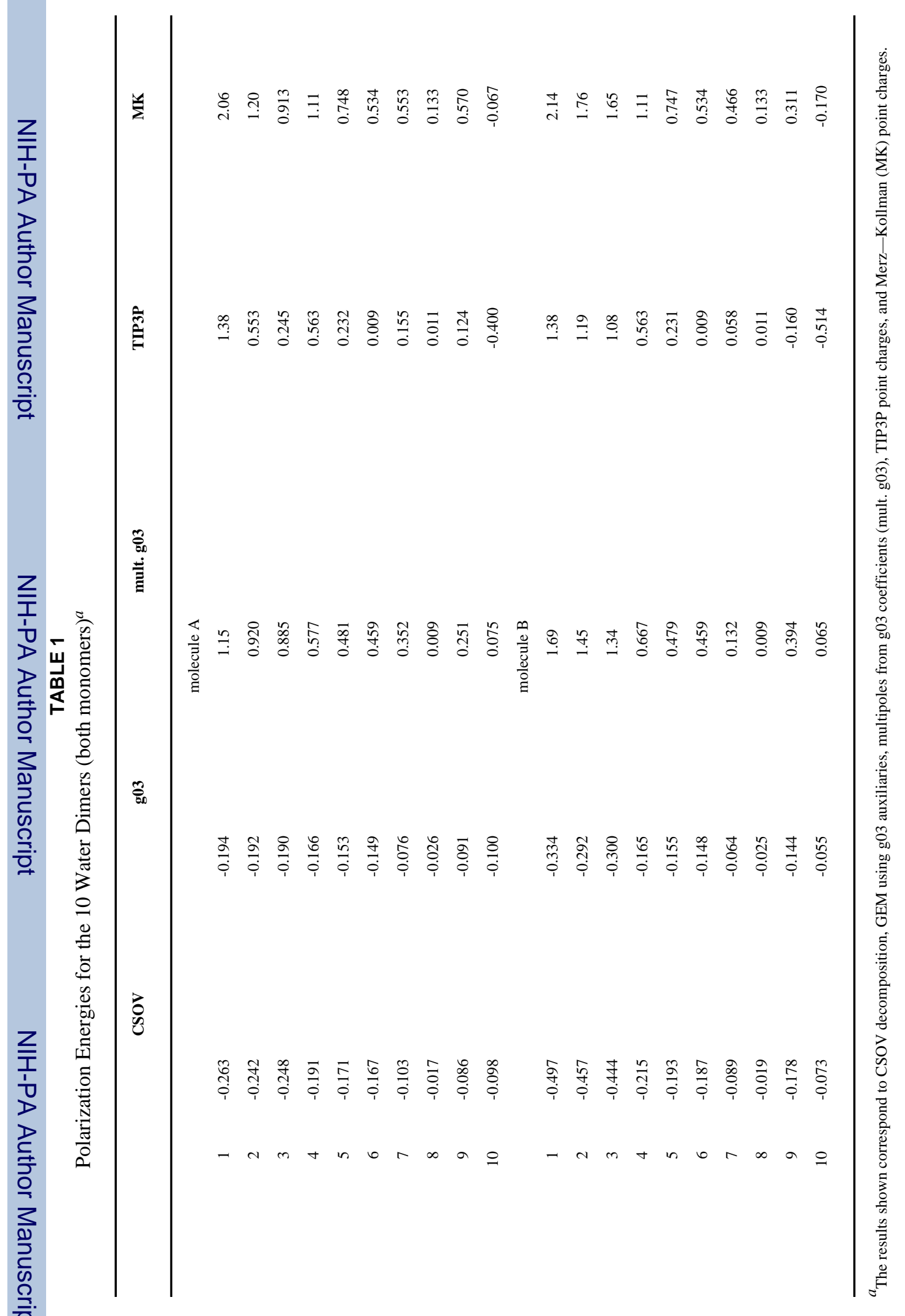

